\title{
A Combinatorial Strategy for Tageting Papillary Thyroid Carcinoma with MEK Inhibitor and SHP2 Inhibitor
}

\section{Jingtai Zhi}

Tianjin Medical University Cancer Institute and Hospital: Tianjin Tumor Hospital Jiaoyu Yi

Tianjin Medical University Cancer Institute and Hospital: Tianjin Tumor Hospital

\section{Xiukun Hou}

Tianjin Medical University Cancer Institute and Hospital: Tianjin Tumor Hospital

\section{Wei Wang}

Tianjin First Central Hospital

\section{Weiwei Yang}

Tianjin First Central Hospital

\section{Linfei Hu}

Tianjin Medical University Cancer Institute and Hospital: Tianjin Tumor Hospital Jianfeng Huang

Sanford Burnham Prebys Medical Discovery Institute

\section{Shicheng Guo}

University of Wisconsin-Madison

\section{Xianhui Ruan}

Tianjin Medical University Cancer Institute and Hospital: Tianjin Tumor Hospital

\section{Xiangqian Zheng}

Tianjin Medical University Cancer Institute and Hospital: Tianjin Tumor Hospital Ming Gao ( $\square$ headandneck2008@126.com )

Tianjin Medical University Cancer Institute and Hospital: Tianjin Tumor Hospital https://orcid.org/0000-0002-2743-2247

\section{Research}

Keywords: Papillary thyroid carcinoma, MEK inhibitor resistance, SHP2, combination strategy

Posted Date: October 8th, 2020

DOI: https://doi.org/10.21203/rs.3.rs-86062/v1 
License: (c) (i) This work is licensed under a Creative Commons Attribution 4.0 International License. Read Full License 


\section{Abstract}

Background: Pharmacologic targeting of components of MAPK/ERK pathway in thyroid carcinoma is often limited due to the development of adaptive resistance. However, the detailed mechanism for MEK inhibitor (MEKi) resistance is not fully understood in papillary thyroid carcinoma (PTC).

Methods: RNA-seq was performed in MEKi-resistant PTC cell lines (K1, BCPAP, TPC-1 and KTC-1) to investigate the intrinsic mechanism of drug resistance. Colony formation assay, cell viability assay, cell cycle analysis and various murine models, including xenograft model, long-term MEKi-treated model, and transgenic model were conducted to evaluate the treatment effect of combination therapy (SHP099 and selumetinib).

Results: Multiple receptor tyrosine kinases (RTKs) signaling pathways as well as Src-homology 2 domaincontaining phosphatase 2 (SHP2) were activated in MEKi-resistant cells. Given the physiological role of SHP2 as the downstream of many RTKs, we first found that blockage of SHP2 abrogated MEKi resistance in thyroid cancer. Interestingly, we also found MEKi in combination with SHP2 inhibitor remarkably suppress rebound of MEK/ERK pathway compared to that of MEKi treatment alone, which significantly improved antitumor effects of MEKi. Various murine models confirmed the synergistic suppression on PTC in mice treated with both inhibitors.

Conclusion: SHP2 blockade by SHP099 in combination with selumetinib is a promising therapeutic approach for advanced thyroid cancer.

\section{Background}

Despite the promising overall prognosis of differentiated thyroid cancer (DTC), $5-10 \%$ of patients continue to suffer from advanced and treatment-refractory disease after standard therapy ${ }^{[1-3]}$. Once progression emerges, surgery, external beam radiation, watchful waiting and experimental trials would be sequentially performed, and these usually have marginal survival benefits ${ }^{[4,5]}$. Patients with advanced DTC only have a $10 \%$ survival rate, in contrast to approximately $98 \%$ of patients with the indolent one ${ }^{[6}$, 7]. Hence, there is an urgent need to develop novel reliable treatment strategies for advanced DTC.

ERK signaling is the most commonly affected in DTC, and is widely correlated with cell proliferation, differentiation, gene transcription and immune escap $\mathrm{e}^{[8,9]}$. Impairing the ERK signaling is the primary strategy for targeted therapy, and mainly occurs at the levels of BRAF and MEK ${ }^{[8,10]}$. Supported by the encouraging preclinical data, MEK inhibitor (MEKi) selumetinib has been approved for the treatment of advanced DTC ${ }^{[11]}$. However, there are still two limitations for selumetinib in the process of its clinical application. Firstly and most importantly, the emergence of MEKi-resistance is common. Despite initial promising results, a lasting response to MEKi is, however, infrequently seen ${ }^{[8]}$. Furthermore, MEKi has less effect on thyroid cancer cells that lack the BRAF ${ }^{\mathrm{V} 600 \mathrm{E}}$ mutation ${ }^{[12,13]}$. Therefore, it is worthwhile to explore combination strategies that can abolish the intrinsic resistance, and extend the application range. 
Reactivated ERK signaling reportedly appears in various tumor cells after MEKi treatment ${ }^{[14]}$. ERK rebound typically occurs via the activation of different receptor tyrosine kinases (RTKs), even in tumors of the same histotype, making the combination of MEK inhibitors and RTK inhibitors challenging ${ }^{[15,16]}$. The protein-tyrosine phosphatase SHP2 (PTPN11) is a signal-enhancing transducer that acts between RTKs and ERK signaling, and is considered as a novel therapeutic target ${ }^{[17,18]}$.

In the present study, we constructed drug-resistant PTC cell clones with continuous MEKi chemotherapy drug stimulation (selumetinib) and then applied RNA-sequencing to identify resistant related genes and pathways. We found multiple RTKs were activated in MEKi-resistant models and p-ERK was re-activated in PTC cell lines. We demonstrated the combination (MEKi and SHP2i) strategy showed superior antitumor activity than SHP099 or selumetinib alone in the established MEKi-resistant models. Consistent results were also observed in PTC cells harboring the BRAF mutation or RET activation.

\section{Methods}

\section{Cells and drugs}

The K1 and BCPAP cell lines were purchased from Guangzhou Cellcook Biotech Co. (Guangzhou, China). The TPC-1 and KTC-1 cell lines were purchased from the Chinese Academy of Science (Shanghai, China). All cell lines were identified by short tandem repeat (STR) analysis, and cultured in RPMI 1640 (Gibco) supplemented with $10 \%$ fetal bovine serum, penicillin/streptomycin $(5,000$ units $/ \mathrm{mL}$, Gibco) and Iglutamine ( $2 \mathrm{mM}, \mathrm{Gibco})$. The passage number of cells used for the experiments was approximately 2030. All cell lines were tested for mycoplasma contamination. Selumetinib (selleck) and SHP099 (selleck, a potent, selective, orally available SHP2 inhibitor) were used in the present study. The lentivirus containing the SHP2 shRNA plasmid was purchased from Genechem Co. (Shanghai, China).

\section{RNA-Sequence Analysis (RNA-Seq)}

RNA was extracted from PTC cell lines BCPAP and its MEKi-resistant models BCPAP-R. The samples were then processed for RNA-sequencing using the NuGen Ovation Human FFPE RNA-Seq Multiplex System. Total RNA from biological triplicates (4 MEKi-resistant cell clones and 4 original cell clones) was isolated, quality was determined (Bioanalyzer), rRNA was depleted with RiboMinus, multiplexed paired-end libraries were prepared with Illumina TruSeq, and sequencing was performed on an Illumina HiSeq. Quality of the sequencing was determined by running FastQC. GSEA analysis was performance to identify enriched pathway and activiated network modules. All the data have been deposited to GEO/SRA with access id: doi:10.5061/dryad.1zcrjdfqh.

\section{Cell viability assay}


Cells were seeded into a 96 -well plate at 500 cells per well. After incubation for 24 hours, these cells were treated with DMSO, SHP099 $(10 \mu \mathrm{M})$, selumetinib (Sel, $1 \mu \mathrm{M})$, or the inhibitor combination (Comb) at various drug concentrations. After the indicated period of time, these cells were incubated with the CCK8 substrates $(5 \mathrm{mg} / \mathrm{mL})$ for two hours. The optical density was measured at $450 \mathrm{~nm}$.

\section{Colony formation assay}

Cells were seeded into a 24-well plate at 200 cells per well. After 24 hours, these cells were cultured with the indicated inhibitors for two weeks. Then, these cells were stained with $0.05 \%$ crystal violet before photography.

\section{Cell cycle analysis}

The treated cells were fixed in cold $70 \%$ ethanol, and incubated at $4^{\circ} \mathrm{C}$ overnight. After incubation, these cells were resuspended in PBS buffer supplemented with $60 \mu \mathrm{g} / \mathrm{mL}$ of RNase $A$ and $25 \mu \mathrm{g} / \mathrm{mL}$ of propidium iodide $(\mathrm{PI})$ for 15 minutes in the dark at room temperature. Then, the samples were analyzed using a FACS Calibur flow cytometer (BD Biosciences).

\section{Western blot and RTK arrays}

Cells were lysed in modified RIPA buffer containing 1\% PMSF. Equal amounts of total protein were resolved by SDS-PAGE, and transferred onto PVDF membranes (Millipore). Then, these membranes were immunoblotted overnight with the primary antibodies. The primary antibodies used for the western blot were $\mathrm{p}-\mathrm{MEK}$, MEK, p-ERK (Cell Signaling Technology, 4370), ERK (Cell Signaling Technology, 4695), SHP2 (ABCAM, ab32083), p-SHP2 (ABCAM, ab62322), and GAPDH (Cell Signaling Technology, 5174). The human phospho-RTK arrays were purchased from R\&D Systems (ARY001B), and were used according to manufacturer's guidelines.

\section{Animal experiments}

All animal experiments were approved by the Tianjin Medical University Cancer Institute and the Hospital Animal Care and Use Committee, and these were performed according to the IACUC protocol. The thyroid cancer cell line xenografts were established through the subcutaneous injection of $1 \times 10^{5}$ cells into 4week-old male NSG mice. When the tumor volumes reached approximately $15 \mathrm{~mm}^{3}$, these animals were randomly assigned into four groups: first group (Ctrl), mice were orally treated with DMSO, q.d, second group (SHP099), mice were orally treated with $50 \mathrm{mg} / \mathrm{kg}$ of SHP099, q.o.d; third group (Sel), mice received daily oral doses of selumetinib, $20 \mathrm{mg} / \mathrm{kg}$; last group (Comb), mice were treated with the combination therapy. In order to establish the drug resistance model in vivo, ten mice with similar-volume PTC xenografts received oral doses of selumetinib, at $20 \mathrm{mg} / \mathrm{kg}$, once daily. After 15 or 40 days, representative 
xenografts in two groups were picked for the human phospho-RTK arrays. Remaining xenograft from 40 days treated mice was cut into equal sections, and planted into other mice. After seven days, planted animals were randomly assigned into four groups, and treated as described above. At the indicated time points, these animals were sacrificed, and the tumors were excised for further analysis. A transgenic mouse model of spontaneous PTC was established as previous described. In our conditions, the mice spontaneously developed PTC at 6-12 weeks of age. According to weight, six-week-old TPO-Cre BrafCA mice were randomly assigned into four groups: first group (Ctrl), mice were orally treated with DMSO, q.d; second group (SHP099), mice were orally treated with $50 \mathrm{mg} / \mathrm{kg}$ of SHP099, q.o.d, third group (Sel), mice received daily oral doses of selumetinib, $20 \mathrm{mg} / \mathrm{kg}$; last group (Comb), mice were treated with the combination therapy.

\section{Immunohistochemistry (IHC)}

$\mathrm{IHC}$ was performed according to standard protocols. The primary antibodies used for the IHC assays were p-ERK (Cell Signaling Technology, 4370) and Ki67 (Cell Signaling Technology, 9027). The stained slides were independently examined by two pathologists, who were blinded to the treatment information. Hematoxylin and eosin staining were performed by the Department of Pathology of Tianjin Medical University Cancer Institute and Hospital.

\section{RT-PCR}

The RT-PCR assays were performed as previously described ${ }^{[17]}$. The primers were listed as Table 1.

Table 1

The primers of RT-PCR assays.

\begin{tabular}{|lll|}
\hline & FORWARD & REVERSE \\
\hline ETV1 & CTTAGCCGTTCACTCCGCTAT & TCTGTCTTCAGCAGTGGACG \\
\hline ETV4 & GCCCATTTCATTGCCTGGAC & TACACGTAACGCTCACCAGC \\
\hline ETV5 & TAGAACCGGAAGAGGTTGCTC & TTATCCGGGAAAGCCATGGAG \\
\hline FOSL1 & CAGCCCAGCAGAAGTTCCA & ACTGAGGGTAGGTCAGAGGC \\
\hline
\end{tabular}

\section{Statistical analysis}

Data were represented as mean $\pm S D$ of three independent experiments. The statistical analysis was performed using SPSS (IBM Corporation, Armonk, NY, USA) and GraphPad Prism 8.0 software (La Jolla, CA, USA). T tests and ANOVA tests were used for determining statistically significant difference $\left({ }^{\star} P<0.05\right.$, $\star \star P<0.01$, $* * * P<0.001$, compared with Ctrl, @P<0.05, @@P<0.01, @@@P<0.001, compared with Comb) between different inhibitor treated and its control conditions. 


\section{Results}

\section{SHP2 is a potential therapeutic target for selumetinib resistant PTC cell lines.}

In order to verify the intrinsic mechanism for MEKi-resistance, the resistant models were constructed in vitro with BCPAP and TPC-1. Cells were cultured in increasing concentrations of selumetinib until drugresistant clones were more than 10 times less sensitive to selumetinib than their parental lines, termed BCPAP-R and TPC-1-R, respectively (Supplementary Fig. S1a). Colony formation assay further confirmed that the MEKi-resistant cell lines exhibited a significant resistance $(P<0.05)$ than the primary cell lines, after treatment with selumetinib (Supplementary Fig. S1b). Then we performed RNA-sequencing analysis to BCPAP $(N=4)$ and BCPAP-R cells $(N=4)$, RNA-seq identified 4,270 mRNAs with significant change in BCPAP-R relative to BCPAP (Supplementary Fig. S2a and S2b). Based on the total identified mRNAs, GSEA analysis revealed that mRNA of RTKs (including VEGFA, VEGF, IGF1 and EGFR) signaling significantly increased in BCPAP-R (Fig. 1A and 1B). These above results indicated that RTK-induced ERK reaction played the main role in acquired MEKi-resistance. SHP2 is a positive RTK downstream signal transducer, which is recently regarded as a novel target for RTKs-driven cancers ${ }^{[18]}$. Expression of p-SHP2 in both primary and MEKi-resistant cell lines was determined by Western blot. Compared with parental cell lines respectively, p-SHP2 expression was significantly upregulated in BCPAP-R and TPC-1-R. At the same time, $\mathrm{p}$-MEK and $\mathrm{p}$-ERK expression were upregulated continuously, not as the negative feedback inhibition in primary cell lines (Fig. 1C). In order to verify whether SHP2 contributes to MEKi-resistance, two MEKi-resistant cell lines (BCPAP-R and TPC-1-R) were treated with DMSO (Ctrl), SHP099, selumetinib (Sel), or the SHP099/selumetinib combination (Comb), respectively. All examined MEKi-resistant cell lines demonstrated susceptibility to SHP099 with suppressed cell colony formation and viability (Fig. 1D and 1E). Cell cycle analysis revealed that SHP099 arrested MEKi-resistant cell lines at the G1/S phase (Fig. 1F). The co-administration with selumetinib exhibited an increase in efficacy, with additive synergy. SHP099 resensitized BCPAP-R and TPC-1-R to selumetinib, and the combination strategy suppressed the cell colony formation, growth, and cell cycle progression more effectively than either SHP099 or selumetinib alone (Fig. 1D, 1E and 1F). These data indicated that the SHP2-transduced RTKs activation was a dominant mechanism for the MEKi-resistant phenotype, which was synergistically reversed by SHP2 inhibitor SHP099.

\section{The combination strategy suppresses the long-term MEKi- treated tumor growth in vivo}

In order to test the effectiveness of the combined strategy to the long-term MEKi-treated murine model in vivo, mice with $\mathrm{K} 1$ xenografts were established and treated as the flow chart indicated (Fig. 2A). Ten mice with similar-volume PTC xenografts were divided into two groups and received oral doses of selumetinib (20 mg/kg, q.d) for 15 or 40 days, respectively. During the 40 days selumetinib treatment, the tumor 
growth rate was suppressed in approximately 25 days, and this rapidly climbed in approximately 25-40 days (Supplementary Fig. S3). Representative xenografts treated with selumetinib for 15 or 40 days were selected for the phospho-RTK array, which revealed that multiple RTKs were consistently activated after 40 days MEKi-treatment (Fig. 2B). In order to analyze the efficacy of the combined strategy to RTKactivated xenografts, the 40 days MEKi-treated xenograft remains were cut into equal sections, and planted into other mice. After seven days, planted mice were randomly assigned into four groups, and treated with DMSO (Ctrl), SHP099, selumetinib (Sel) and both drugs combination (Comb) for 15 days, respectively. Selumetinib had minimal effects on RTK-activated tumors, demonstrating that the tumor models had partial resistance to MEKi. The upregulated susceptibility to SHP099 in long-term MEKitreated tumors indicated that activated RTKs played a major role in PTC cells proliferation. Of note, the SHP099/selumetinib combination caused tumor shrinkage more effectively than the single agent (Fig. 2C, 2D and 2E), demonstrating that developed MEKi-resistance depending on SHP2. As revealed by the IHC assay (Fig. 2F), the SHP099/selumetinib combination treatment group had a lower p-ERK expression, and fewer Ki67-positive cells. Stable weight, normal behavior and appeared health were showed in MEKiresistant models after combination treatment (Supplementary Fig. S4). These data suggested that the combined SHP2/MEK inhibition continued to be effective for suppressing tumor growth in the long-term MEKi-treated models in vivo.

\section{Suppression of SHP2 abrogates the RTKs-induced MEK/ERK pathway rebound}

After treatment with MAPK inhibitor, various cancer models can develop ERK signaling rebound induced by RTKs ${ }^{[19-22]}$. In order to examine whether the ERK rebound existed in PTCs after treatment with MEKi, the $p$-ERK expression was detected after 0, 1, 24 and 48 hours of selumetinib treatment. Western blot revealed that the $p$-ERK expression initially declined after one hour, and rapidly rebounded to the baseline or to an even higher level at 48 hours (Fig. 3A). In order to verify the relationship between ERK signaling rebound and activated RTKs in PTCs, a human phospho-RTK array was performed to determine the RTK activation status. As expected, the phosphorylation levels of several RTKs were upregulated in K1 after MEKi-treatment (Fig. 3B). Activated by RTKs, p-SHP2 expression significantly increased $(P<0.001)$ in all four cell lines treated with selumetinib (Fig. 3C). Hence, it was hypothesized that therapy that co-targets MEK and SHP2 could effectively abolish the ERK signaling reactivation. For further confirmation, four PTC cell lines were treated with DMSO, SHP099 alone, selumetinib alone, or the SHP099/selumetinib combination, and the change in p-ERK expression within 48 hours was detected. Compared with SHP099 or selumetinib alone, the combination of SHP099 and selumetinib persistently inhibited the p-ERK expression (Fig. 3D). As measured by ETV1, 4, 5, and FOSL1 mRNA levels, ERK dependent transcription was also significantly inhibited $(P<0.01)$ by combination strategy (Fig. 3E). In addition, the combination of MEKi and SHP2 knockdown had similar effects to the SHP099/MEKi treatment, indicating that SHP099 is "on-target" in PTC cell lines (Fig. 3F, $\mathbf{3 G}$ and $\mathbf{3 H}$ ). Taken together, these data demonstrated that 


\section{SHP2 inhibition combined with selumetinib inhibits cell progression in PTC in vitro}

In order to determine whether the combined SHP2/MEK inhibition could be more effective in PTC cell lines, colony formation and viability (CCK8) assays were further performed (Fig. 4A and 4B). As a single agent, SHP099 had a variable effect on colony formation in PTC cell lines with different genetic backgrounds. A minimal effect was detected in three BRAF mutation cell lines (which was consistent with previous studies), while a remarkable effect $(P<0.01)$ was observed in the BRAF wild-type harboring the RET fusion mutation. However, compared with SHP099 or selumetinib treatment alone, few or no detectable colonies in all PTCs were found after SHP099/selumetinib combination treatment $(P<0.01)$. Similar effects were observed in cell viability assays. The combination resulted in intense growth inhibition $(P<0.01)$ in all PTCs. Given the SHP099/selumetinib combination effect on PTC cell lines, it was determined whether the combination treatment suppressed the cell proliferation by regulating cell cycle progression (Fig. 4C). The SHP099/selumetinib combination arrested PTC cell lines at the G1/S phase. Same as the previous study to indicate that SHP099 is "on-target" in PTC cell lines, SHP2 knockdown had similar effects to the SHP099/MEKi treatment (Fig. 4E and 4F). These data suggested that the combined SHP2/MEK inhibition could inhibit PTC cell proliferation powerfully in vitro.

\section{The combination of SHP099 and selumetinib is a promising therapeutic approach in vivo}

To confirm our results in models in vivo, mice carrying K1 or TPC-1 xenografts were utilized to examine the antitumor activity of MEK/SHP2 inhibition. Compared with mice treated with SHP099 or selumetinib treatment alone, mice in the combination group markedly reduced tumor volume and weight (Fig. 5A, 5B and 5C), and more effectively suppressed p-ERK level (Fig. 5D). Xenografts isolated from combinationtreated mice had fewer proliferating cells than those derived from other mice, as measured by Ki67 staining (Fig. 5D). The single-agent effects were similar to the above results in vitro. After treatment with combination strategy, stable weight, appeared healthy and normal behavior and were showed in all mice carrying K1 or TPC-1 xenografts (Supplementary Fig. S5a and S5b).

We further confirmed our results in a transgenic murine model of spontaneous thyroid cancer (Fig. 6A). Mice treated with SHP099 or selumetinib treatment alone had reduced tumor volume to about $40-50 \%$ of the initial volume, though the combination of SHP099 and selumetinib produced a greater reduction in tumor volume and weight than either therapy alone (Fig. 6B and $6 \mathrm{C}$ ). IHC assay revealed that the SHP099/selumetinib combination treatment group had a lower p-ERK expression, and fewer Ki67-positive 
cells (Fig. 6D). These data suggested that the combined SHP2/MEK inhibition could serve as a powerful therapeutic approach in PTCs, in which single-agent targeted therapeutics usually have a limited effect.

\section{Discussion}

In addition to RTKs reactivation, tumors evade the long-term MEK blockage therapies via various resistance mechanisms ${ }^{[23]}$, such as the BRAF gene amplification in colorectal cancers harboring $\mathrm{BRAF}^{\mathrm{V} 600 \mathrm{E}[24]}$, the increased formation of Raf-1/B-Raf dimers in melanoma cells ${ }^{[25]}$, and the enhanced activation of the PI3K/AKT pathway in melanoma cells ${ }^{[26]}$. Upregulated multiple RTKs signaling was revealed by RNA-seq in MEKi-resistant model in vitro and identified by RTK assay in long-term MEKitreated models in vivo. Activated by RTKs, SHP2 interacts with Ras, participating in signal transducer and activator of MAPK pathway to promote tumor progression [27]. A higher SHP2 activity was found in all resistant clones, which indicated that SHP2 can be activated by RTKs, and plays a major role in MEKiresistance. The combination of selumetinib and SHP099 significantly blocks the tumor growth and ERK activation in all MEKi-resistant models, demonstrating that PTCs with either BRAF ${ }^{\mathrm{V} 600 \mathrm{E}}$ mutation or RET activation acquire MEKi-resistance through activated RTKs, and this mainly depended on SHP2. Hence, the combination strategy may be a promising treatment approach for MEKi-resistant PTC. A schematic model summarizing the mechanism of the SHP099/selumetinib combination strategy in PTC was presented in (Supplementary Fig. S6).

Although a synergy has been widely observed in various tumor types, the combination of SHP099 and selumetinib has been reported to be less effective for certain tumors. RAS is the direct downstream signal for SHP2, and the GTPase activity of different RAS mutants positively correlated with the sensitivity to SHP099. Certain RAS mutations (G13D and Q61X) have the lowest intrinsic GTPase activity than other mutations, thereby emerging the resistance to this combination ${ }^{[28-30]}$. Most RTKs reactivate the RAS/MEK/ERK pathway through SHP2 to form adaptive resistance against MAPK signaling inhibitors, while remaining RTKs, including FGFR, could transduce signals in a SHP2-independent fashion [22]. Depend on FGFR activation, a subset of ATC cells harboring BRAF ${ }^{\mathrm{V} 600 \mathrm{E}}$ has been proven to be insensitive to the combination of SHP099 and MAPK inhibitor [22]. However, although derived from thyroid follicular cells as well, our study demonstrated SHP2/MEK inhibition effectively suppressed tumor progression in all tested PTC models both in vivo and in vitro. In addition, RTK assay also revealed that MEKi remarkably induced SHP2-dependent RTKs activation in PTC models. Hence, distinct with ATC, the combination of SHP099 and selumetinib is a promising therapeutic approach for PTC.

RET is an RTK involved in the development of PTC and medullary thyroid carcinomas (MTC) ${ }^{[31,32]}$. RET/PTC fusions were presented in PTC, and these can reach $50-70 \%$ in pediatric patients, and in cases that have experienced radioiodine exposure ${ }^{[33]}$. In contrast with the PTC cell line harboring BRAFV600E mutation, high SHP2 activity and SHP099 sensitivity were found in TPC-1, a PTC cell line harboring RET Gain-of-function fusion. Resemble trend also occurred in TT (Supplementary Fig. S7a and S7b), a MTC cell line harboring constitutive-activation RET $^{\mathrm{C} 634 \mathrm{~W}}$ mutation ${ }^{[34]}$. Above data suggested that SHP099 
significantly suppresses RET activation-driven PTC progression, though SHP2/MEK blockage produced a greater inhibition in tumor development.

The present study has several limitations. First, the effect of the combination of SHP099 and selumetinib in PTC cell lines harboring RAS mutations was not evaluated. This was limited by the low mutation rate of RAS in PTCs ${ }^{[35]}$, and the well-known relationship between RAS mutation and the SHP099 effect ${ }^{[30,36 \text {, }}$ 37]. Second, the impact of the combination strategy on the microenvironment of PTC could not be evaluated. Both SHP099 and selumetinib have been reported to be correlated with various immune cell activations ${ }^{[31,38,39]}$. Our study revealed that SHP099 can effectively suppressed the tumor growth in the immunocompetent transgenic mouse model of spontaneous PTC, which was consistent with the above studies. Hence, further studies are needed to determine the synergy of SHP099 and selumetinib in shaping the microenvironment of PTCs.

\section{Conclusions}

In conclusion, the combination of SHP099 and selumetinib abolished the RTK-mediated ERK rebound, resulting in growth inhibition. Compared with the use of SHP099 or selumetinib alone, the combination strategy overcame the intrinsic resistance in the long-term and extended the application range in MAPKactivated PTCs more effectively. The combination of SHP2 inhibitor SHP099 and selumetinib may be a promising and meaningful therapeutic strategy against PTC.

\section{Abbreviations}

MEKi: MEK inhibitor

PTC: papillary thyroid carcinoma

RTKs: receptor tyrosine kinases

SHP2: Src-homology 2 domain-containing phosphatase 2

DTC: differentiated thyroid cancer

SHP2i: Src-homology 2 domain-containing phosphatase 2 inhibitor

\section{Declarations}

\section{Acknowledgements}

Not applicable.

\section{Authors' contributions}


JZ, JY, XH, XR, WW, WY, LH and SG contributed to manuscript draft and data analysis. JH, XZ and MG critically revised the manuscript. All authors read and approved the final manuscript.

\section{Funding}

This work was partially supported by grants from National Natural Science Foundation of China (Grant No. 81872169), Tianjin Key Research and Development Program Science and Technology Support Key Projects (Grant No. 17YFZCSY00690), and Tianjin Municipal Science and Technology Project (Grant No. 19JCYBJC27400).

\section{Availability of data and materials}

All data in our study are available upon request.

\section{Ethics approval and consent to participate}

Not applicable.

\section{Consent for publication}

All authors of this paper have approved the final version of the manuscript.

\section{Competing interests}

The authors declare that there are no conflicts of interest.

\section{References}

1. Siegel RL, Miller KD. Cancer statistics, 2019. 2019;69(1):7-34.doi:10.3322/caac.21551

2. Lim H, Devesa SS, Sosa JA, Check D, Kitahara CM. Trends in Thyroid Cancer Incidence and Mortality in the United States, 1974-2013. Jama. 2017;317(13):1338-1348.doi:10.1001/jama.2017.2719

3. Cronin KA, Lake AJ, Scott S. Annual Report to the Nation on the Status of Cancer, part I: National cancer statistics. 2018;124(13):2785-2800.doi:10.1002/cncr.31551

4. Carneiro RM, Carneiro BA, Agulnik M, Kopp PA, Giles FJ. Targeted therapies in advanced differentiated thyroid cancer. Cancer treatment reviews. 2015;41(8):690698.doi:10.1016/j.ctrv.2015.06.002

5. Lirov R, Worden FP, Cohen MS. The Treatment of Advanced Thyroid Cancer in the Age of Novel Targeted Therapies. Drugs. 2017;77(7):733-745.doi:10.1007/s40265-017-0733-1 
6. Cooper DS, Doherty GM, Haugen BR, Kloos RT, Lee SL, Mandel SJ, Mazzaferri EL, Mclver B, Pacini F, Schlumberger M, Sherman SI, Steward DL, Tuttle RM. Revised American Thyroid Association management guidelines for patients with thyroid nodules and differentiated thyroid cancer. Thyroid : official journal of the American Thyroid Association. 2009;19(11):1167-

1214.doi:10.1089/thy. 2009.0110

7. Durante C, Haddy N, Baudin E, Leboulleux S, Hartl D, Travagli JP, Caillou B, Ricard M, Lumbroso JD, De Vathaire F, Schlumberger M. Long-term outcome of 444 patients with distant metastases from papillary and follicular thyroid carcinoma: benefits and limits of radioiodine therapy. The Journal of clinical endocrinology and metabolism. 2006;91(8):2892-2899.doi:10.1210/jc.2005-2838

8. Zaballos MA, Acuna-Ruiz A, Morante M, Crespo P, Santisteban P. Regulators of the RAS-ERK pathway as therapeutic targets in thyroid cancer. Endocrine-related cancer. 2019.doi:10.1530/erc-19-0098

9. Varricchi G, Loffredo S. The Immune Landscape of Thyroid Cancer in the Context of Immune Checkpoint Inhibition. 2019;20(16).doi:10.3390/ijms20163934

10. Savoia P, Fava P. Targeting the ERK Signaling Pathway in Melanoma. 2019;20(6).doi:10.3390/ijms20061483

11. Naoum GE, Morkos M, Kim B, Arafat W. Novel targeted therapies and immunotherapy for advanced thyroid cancers. Molecular cancer. 2018;17(1):51.doi:10.1186/s12943-018-0786-0

12. Ball DW, Jin N, Rosen DM, Dackiw A, Sidransky D, Xing M, Nelkin BD. Selective growth inhibition in BRAF mutant thyroid cancer by the mitogen-activated protein kinase kinase 1/2 inhibitor AZD6244. The Journal of clinical endocrinology and metabolism. 2007;92(12):4712-4718.doi:10.1210/jc.20071184

13. Hayes DN, Lucas AS, Tanvetyanon T, Krzyzanowska MK, Chung CH, Murphy BA, Gilbert J, Mehra R, Moore DT, Sheikh A, Hoskins J, Hayward MC, Zhao N, O'Connor W, Weck KE, Cohen RB, Cohen EE. Phase II efficacy and pharmacogenomic study of Selumetinib (AZD6244; ARRY-142886) in iodine131 refractory papillary thyroid carcinoma with or without follicular elements. Clinical cancer research : an official journal of the American Association for Cancer Research. 2012;18(7):20562065.doi:10.1158/1078-0432.ccr-11-0563

14. Johnson GL, Stuhlmiller TJ, Angus SP, Zawistowski JS, Graves LM. Molecular pathways: adaptive kinome reprogramming in response to targeted inhibition of the BRAF-MEK-ERK pathway in cancer. Clinical cancer research : an official journal of the American Association for Cancer Research. 2014;20(10):2516-2522.doi:10.1158/1078-0432.ccr-13-1081

15. Herr R, Halbach S. BRAF inhibition upregulates a variety of receptor tyrosine kinases and their downstream effector Gab2 in colorectal cancer cell lines. 2018;37(12):15761593.doi:10.1038/s41388-017-0063-5

16. Ponz-Sarvise M, Corbo V, Tiriac H, Engle DD, Frese KK, Oni TE, Hwang Cl, Öhlund D, Chio IIC, Baker LA, Filippini D, Wright K, Bapiro TE, Huang P, Smith P, Yu KH, Jodrell DI, Park Y, Tuveson DA. Identification of Resistance Pathways Specific to Malignancy Using Organoid Models of Pancreatic Cancer. 
Clinical cancer research : an official journal of the American Association for Cancer Research. 2019;25(22):6742-6755.doi:10.1158/1078-0432.ccr-19-1398

17. Fedele C, Ran H. SHP2 Inhibition Prevents Adaptive Resistance to MEK Inhibitors in Multiple Cancer Models. 2018;8(10):1237-1249.doi:10.1158/2159-8290.cd-18-0444

18. Zhang J, Zhang F, Niu R. Functions of Shp2 in cancer. Journal of cellular and molecular medicine. 2015;19(9):2075-2083.doi:10.1111/jcmm.12618

19. Amodio V, Yaeger R, Arcella P, Cancelliere C, Lamba S, Lorenzato A, Arena S. EGFR blockade reverts resistance to KRAS G12C inhibition in colorectal cancer. 2020.doi:10.1158/2159-8290.cd-20-0187

20. Rosell R, Karachaliou N, Morales-Espinosa D, Costa C, Molina MA, Sansano I, Gasco A, Viteri S, Massuti B, Wei J, González Cao M, Martínez Bueno A. Adaptive resistance to targeted therapies in cancer. Translational lung cancer research. 2013;2(3):152-159.doi:10.3978/j.issn.22186751.2012 .12 .08

21. Shi H, Kong X, Ribas A, Lo RS. Combinatorial treatments that overcome PDGFR $\beta$-driven resistance of melanoma cells to V600EB-RAF inhibition. Cancer research. 2011;71(15):50675074.doi:10.1158/0008-5472.can-11-0140

22. Ahmed TA, Adamopoulos C, Karoulia Z, Wu X, Sachidanandam R, Aaronson SA, Poulikakos PI. SHP2 Drives Adaptive Resistance to ERK Signaling Inhibition in Molecularly Defined Subsets of ERKDependent Tumors. Cell reports. 2019;26(1):65-78.e65.doi:10.1016/j.celrep.2018.12.013

23. Lake D, Correa SA. Negative feedback regulation of the ERK1/2 MAPK pathway. 2016;73(23):43974413

24. Corcoran RB, Dias-Santagata D, Bergethon K, lafrate AJ, Settleman J, Engelman JA. BRAF gene amplification can promote acquired resistance to MEK inhibitors in cancer cells harboring the BRAF V600E mutation. Science signaling. 2010;3(149):ra84.doi:10.1126/scisignal.2001148

25. Lito P, Pratilas CA, Joseph EW, Tadi M, Halilovic E, Zubrowski M, Huang A, Wong WL, Callahan MK, Merghoub T, Wolchok JD, de Stanchina E, Chandarlapaty S, Poulikakos PI, Fagin JA, Rosen N. Relief of profound feedback inhibition of mitogenic signaling by RAF inhibitors attenuates their activity in BRAFV600E melanomas. Cancer cell. 2012;22(5):668-682.doi:10.1016/j.ccr.2012.10.009

26. Turke AB, Song Y, Costa C, Cook R, Arteaga CL, Asara JM, Engelman JA. MEK inhibition leads to $\mathrm{PI3K} / \mathrm{AKT}$ activation by relieving a negative feedback on ERBB receptors. Cancer research. 2012;72(13):3228-3237.doi:10.1158/0008-5472.can-11-3747

27. Huang WQ, Lin Q, Zhuang X, Cai LL, Ruan RS, Lu ZX, Tzeng CM. Structure, function, and pathogenesis of SHP2 in developmental disorders and tumorigenesis. Current cancer drug targets. 2014;14(6):567-588.doi:10.2174/1568009614666140717105001

28. Lopez-Alberca MP, Nazare M, Reichert M, Saur D, Erkan MM, Hopt UT, Sainz B, Jr., Birchmeier W, Schmid RM, Lesina M, Algul H. Nature medicine.doi:10.1038/s41591-018-0024-8

29. Nichols RJ, Haderk F, Stahlhut C, Schulze CJ, Hemmati G, Wildes D, Tzitzilonis C, Mordec K, Marquez A, Romero J, Hsieh T, Zaman A, Olivas V, McCoach C, Blakely CM, Wang Z, Kiss G, Koltun ES, Gill AL, Singh M, Goldsmith MA, Smith JAM. RAS nucleotide cycling underlies the SHP2 phosphatase 
dependence of mutant BRAF-, NF1- and RAS-driven cancers. 2018;20(9):1064-

1073.doi:10.1038/s41556-018-0169-1

30. Mainardi S, Mulero-Sanchez A, Prahallad A, Germano G, Bosma A, Krimpenfort P, Lieftink C, Steinberg JD, de Wit N. SHP2 is required for growth of KRAS-mutant non-small-cell lung cancer in vivo. 2018;24(7):961-967.doi:10.1038/s41591-018-0023-9

31. Haroon Al Rasheed MR, Xu B. Molecular Alterations in Thyroid Carcinoma. Surgical pathology clinics. 2019;12(4):921-930.doi:10.1016/j.path.2019.08.002

32. de Groot JW, Links TP, Plukker JT, Lips CJ, Hofstra RM. RET as a diagnostic and therapeutic target in sporadic and hereditary endocrine tumors. Endocrine reviews. 2006;27(5):535-

560.doi:10.1210/er.2006-0017

33. Zhu Z, Ciampi R, Nikiforova MN, Gandhi M, Nikiforov YE. Prevalence of RET/PTC rearrangements in thyroid papillary carcinomas: effects of the detection methods and genetic heterogeneity. The Journal of clinical endocrinology and metabolism. 2006;91(9):3603-3610.doi:10.1210/jc.2006-1006

34. Santoro M, Carlomagno F. Central role of RET in thyroid cancer. Cold Spring Harbor perspectives in biology. 2013;5(12):a009233.doi:10.1101/cshperspect.a009233

35. Landa I, Pozdeyev N, Korch C, Marlow LA, Smallridge RC, Copland JA, Henderson YC, Lai SY, Clayman GL, Onoda N, Tan AC, Garcia-Rendueles MER, Knauf JA. Comprehensive Genetic Characterization of Human Thyroid Cancer Cell Lines: A Validated Panel for Preclinical Studies. 2019;25(10):3141-3151.doi:10.1158/1078-0432.ccr-18-2953

36. Valencia-Sama I, Ladumor Y. NRAS status determines sensitivity to SHP2 inhibitor combination therapies targeting the RAS-MAPK pathway in neuroblastoma. 2020.doi:10.1158/0008-5472.can-193822

37. Ruess DA, Heynen GJ, Ciecielski KJ, Ai J, Berninger A, Kabacaoglu D, Gorgulu K. Mutant KRAS-driven cancers depend on PTPN11/SHP2 phosphatase. 2018;24(7):954-960.doi:10.1038/s41591-018-00248

38. Durante C, Puxeddu E, Ferretti E, Morisi R, Moretti S, Bruno R, Barbi F, Avenia N, Scipioni A, Verrienti A, Tosi E, Cavaliere A, Gulino A, Filetti S, Russo D. BRAF mutations in papillary thyroid carcinomas inhibit genes involved in iodine metabolism. The Journal of clinical endocrinology and metabolism. 2007;92(7):2840-2843.doi:10.1210/jc.2006-2707

39. Liu Q, Qu J, Zhao M, Xu Q, Sun Y. Targeting SHP2 as a promising strategy for cancer immunotherapy. Pharmacological research. 2020;152:104595.doi:10.1016/j.phrs.2019.104595

\section{Figures}


A

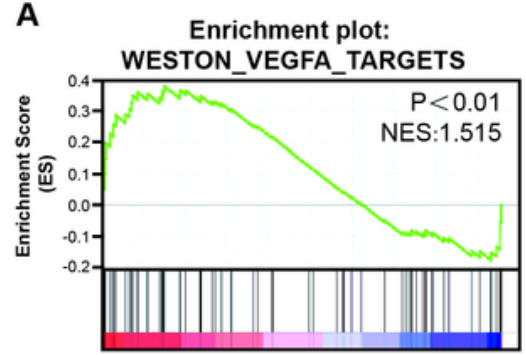

Enrichment plot: KUNINGER_IGF1_VS_PDGFB_TARGETS_UP

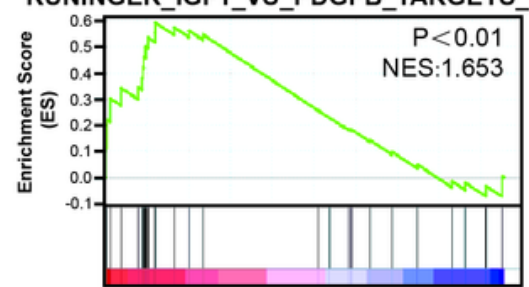

Enrichment plot:

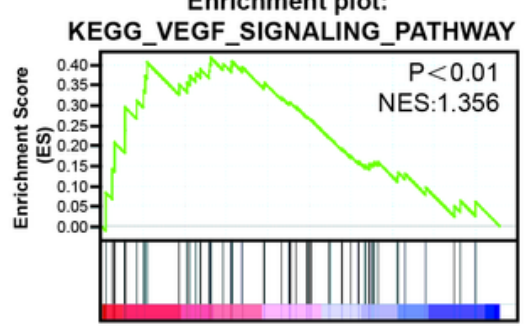

Enrichment plot:

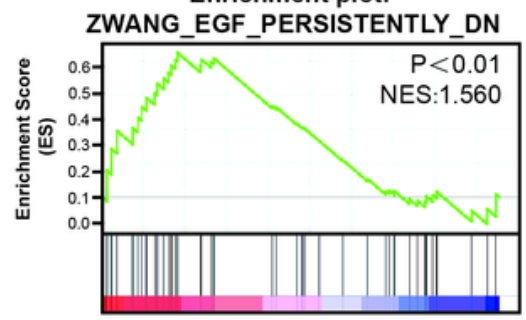

NES: Normalized Enrichment Score
B

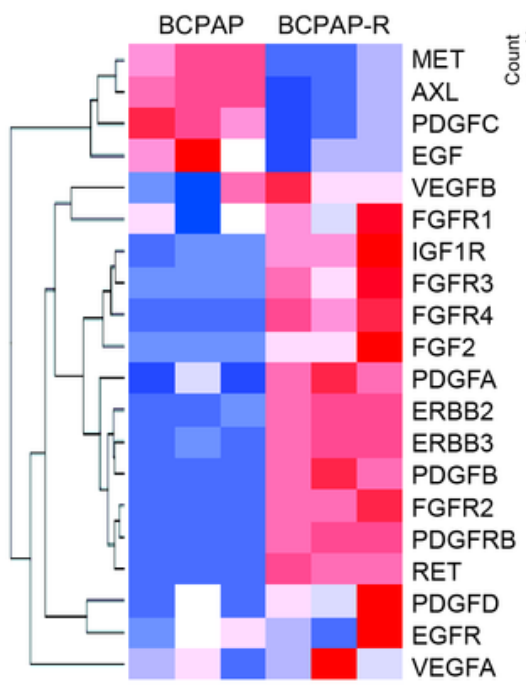

Color Key

and Histogram

and

$10-$

$\int_{-1}^{1} \frac{1}{1}$

C
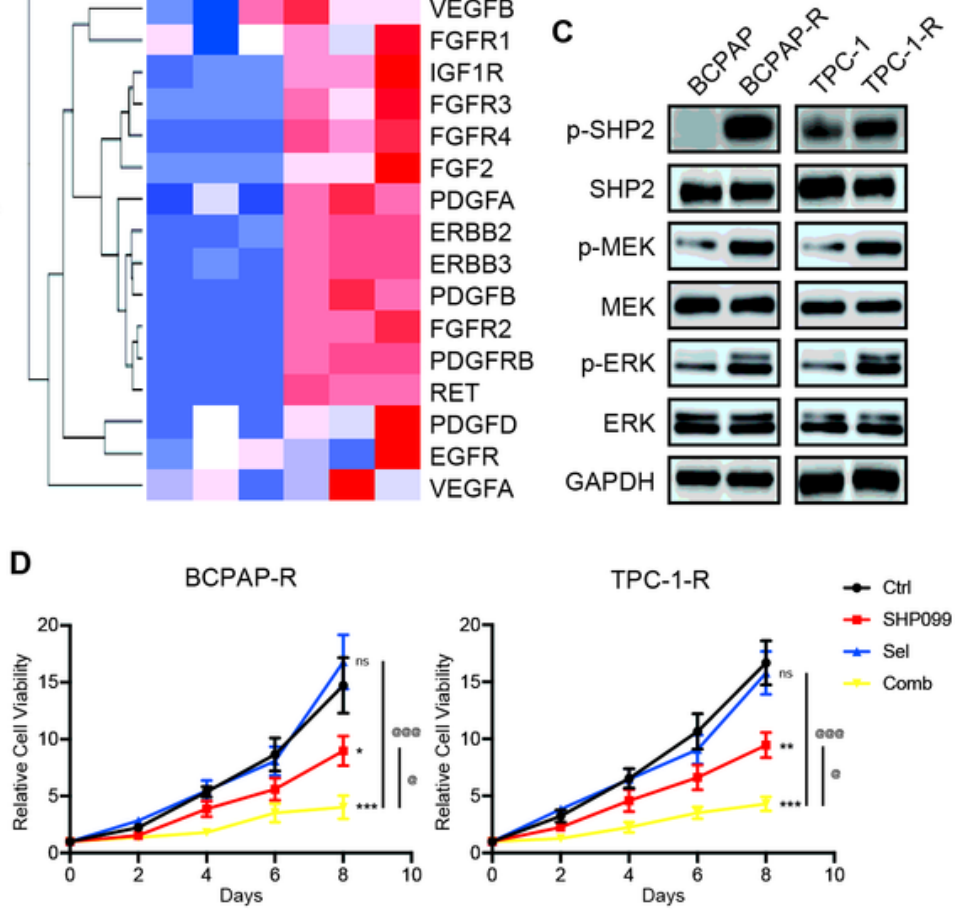

$\mathbf{F}$

BCPAP-R
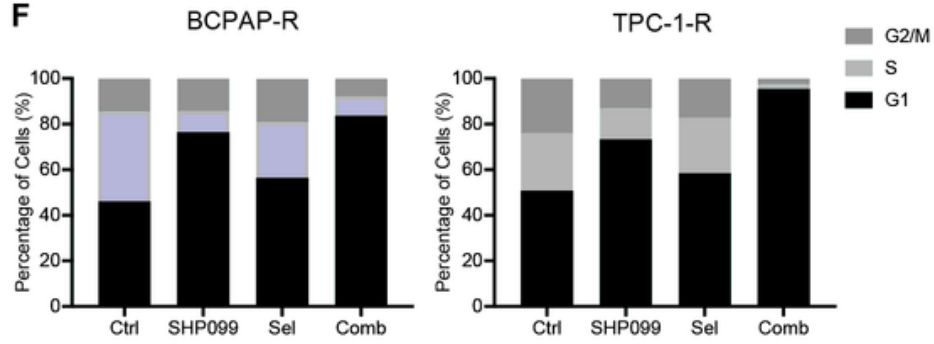

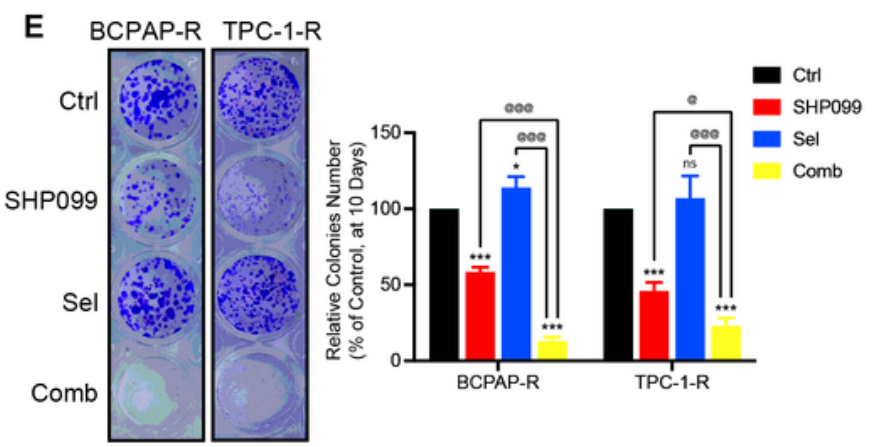

Figure 1

SHP2 is a potential therapeutic target for long-term MEKi-resistant PTC cell lines. (A) GSEA analysis of total identified mRNAs. mRNA of RTKs signaling significantly increased in BCPAP-R. (B) Heatmap of RTKs were up-regulated and represented as a heatmap, with the scale showing fold changes. (C) The SHP2 was significantly activated in the BCPAP-R and TPC-1-R cell lines. Two MEKi-resistant models were treated with DMSO (Ctrl), SHP099 $(10 \mu \mathrm{M})$, selumetinib $(\mathrm{Sel}, 1 \mu \mathrm{M})$, or both drugs (Comb). The cell viability 
(D) and colony formation (E) assays were assessed at 2-8 and 10 days, respectively; ${ }^{\star} P<0.05,{ }^{\star \star} P<0.01$, $\star \star \star P<0.001$, compared with Ctrl, @P $<0.05$, @@P $<0.01$, @@@P<0.001, compared with Comb, ANOVA-test. All data were repeated at least three times independently and represented as mean $\pm S D$. This showed that the SHP099/selumetinib combination group exhibited a more significant effect in inhibiting the proliferation in the long-term MEKi-resistant model than other groups. (F) The SHP099/selumetinib combination significantly induced the G1 phase arrest in the BCPAP-R and TPC-1-R cell lines. The cell cycle distribution was assessed by flow cytometry. The percentage of cells in the G1, S and G2/M phase was plotted. 
A

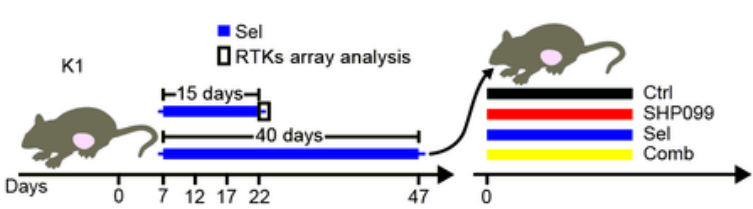

B

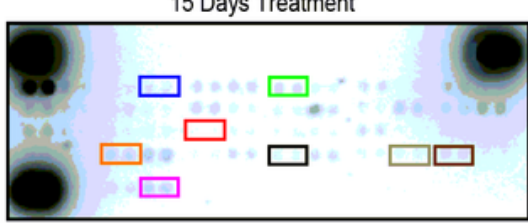

40 Days Treatment
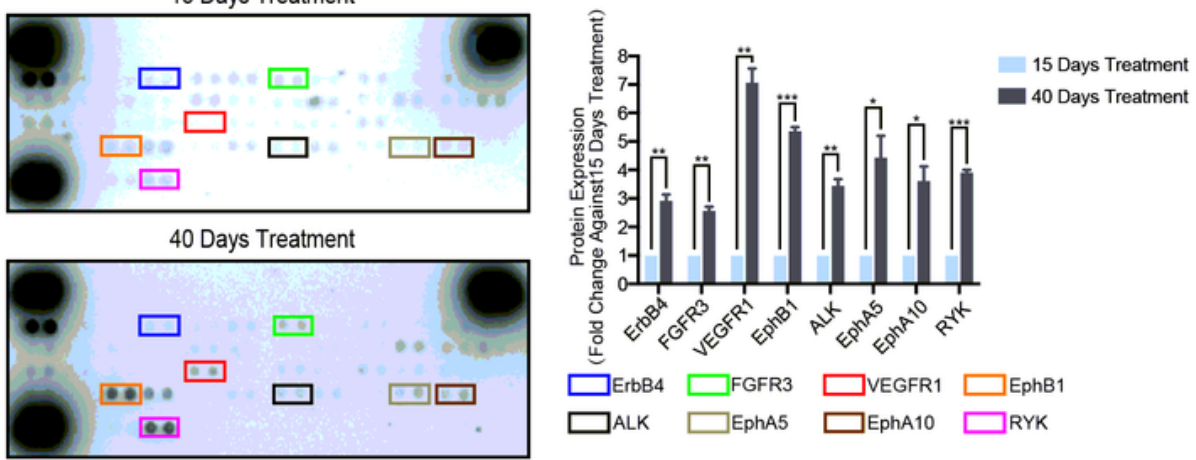

C

D

E
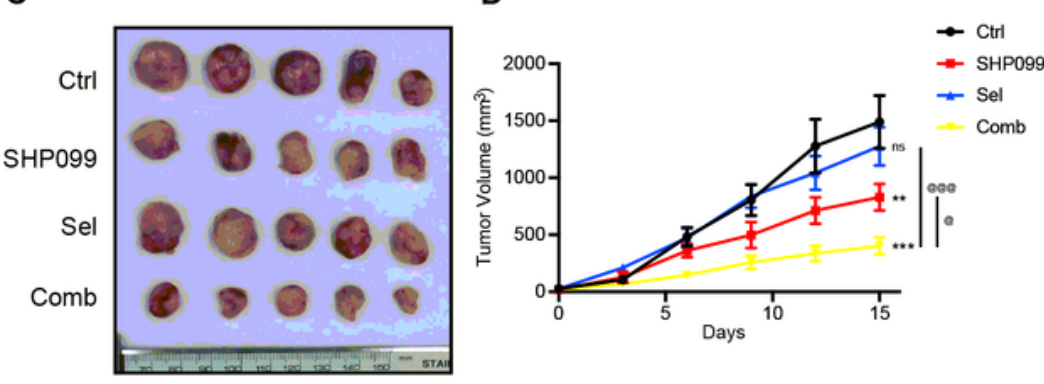

$\mathbf{F}$
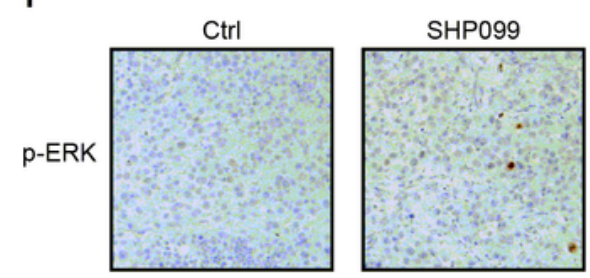

Selumetinib
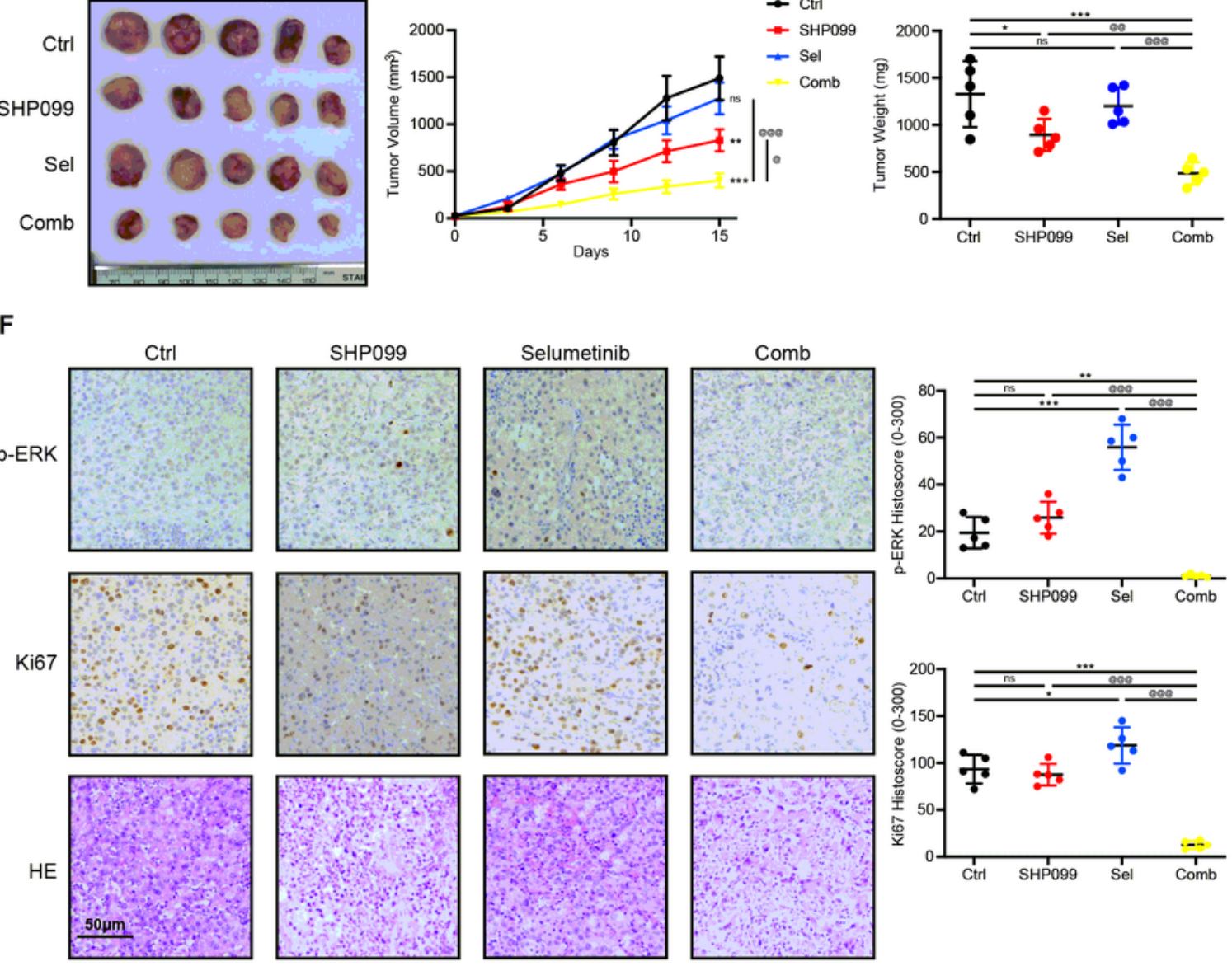

\section{Figure 2}

SHP099 abolished the MEKi-resistance in long-term selumetinib treated murine model. (A) Therapy protocol for mice with K1 xenografts. (B) Phospho-RTK arrays revealed the upregulation of multiple activated RTKs in tumor tissue from mouse with 40 days selumetinib treatment compared with 15 days treatment. ${ }^{*} \mathrm{P}<0.05,{ }^{\star} \times \mathrm{P}<0.01,{ }^{\star} * \star \mathrm{P}<0.001$, two-tailed t-test. (C) The images of the dissected tumors obtained from planted mice in the Ctrl group $(n=5)$, SHP099 group $(n=5)$, selumetinib (Sel) group $(n=5)$ 
and Comb group ( $n=5)$. (D) The growth curve of planted xenografts after treatment with DMSO (Ctrl), SHP099 (50 mg/kg, q.o.d), selumetinib (Sel, $20 \mathrm{mg} / \mathrm{kg}$, q.d) and both drugs (Comb: selumetinib, $20 \mathrm{mg} / \mathrm{kg}$, q.d; SHP099, $50 \mathrm{mg} / \mathrm{kg}$, q.o.d). All planted xenografts volume was measured every five days, until the 15th day. (E) The weight of the dissected tumors from planted mice were analyzed after treatment for 15 days; $n=5$, per group , ${ }^{*}<0.05$, ${ }^{\star *} P<0.01$, ${ }^{* *} P<0.001$, compared with $C$ trl; @P $<0.05$, @@P $<0.01$, @@@P<0.001, compared with Comb, ANOVA. (F) Representative images and QuPath quantitation of pERK and Ki67 immunostaining of xenografts.

A

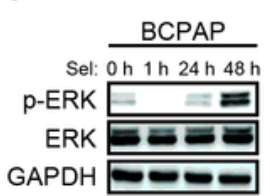

Sel: $\frac{\mathrm{KTC}-1}{0 \mathrm{~h} 1 \mathrm{~h} 24 \mathrm{~h} 48 \mathrm{~h}}$ p-ERK $=-2$

ERK $=2=2$ GAPDH $-2=0$
B

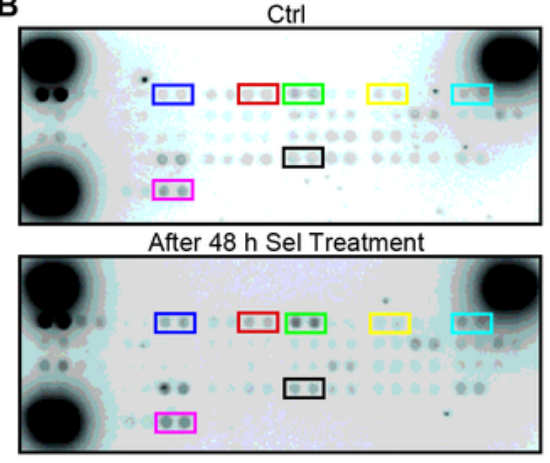

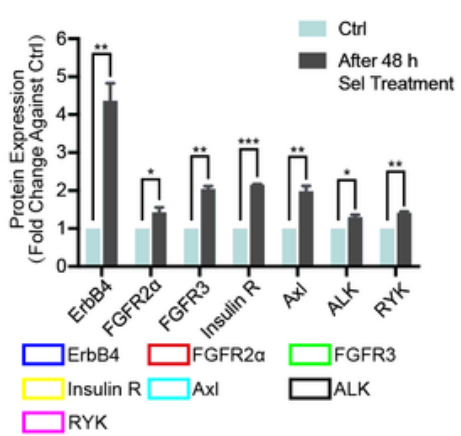

$\square$ RYK
C

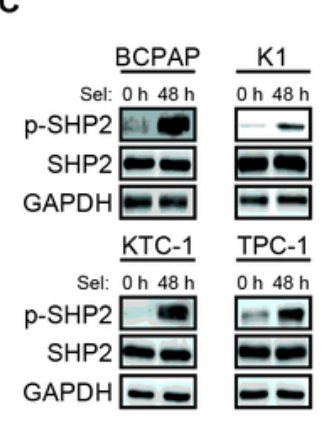

D

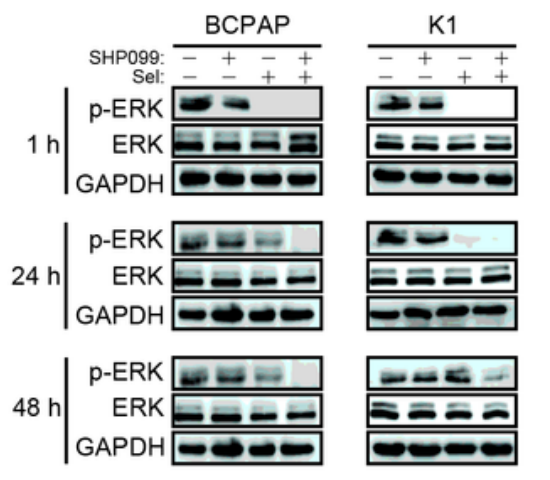

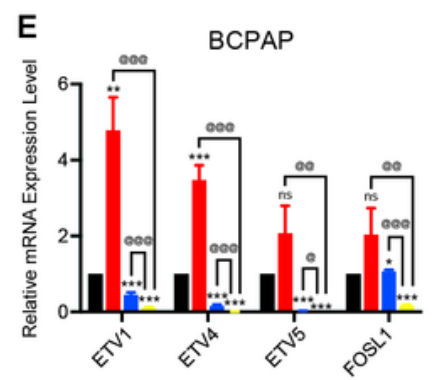
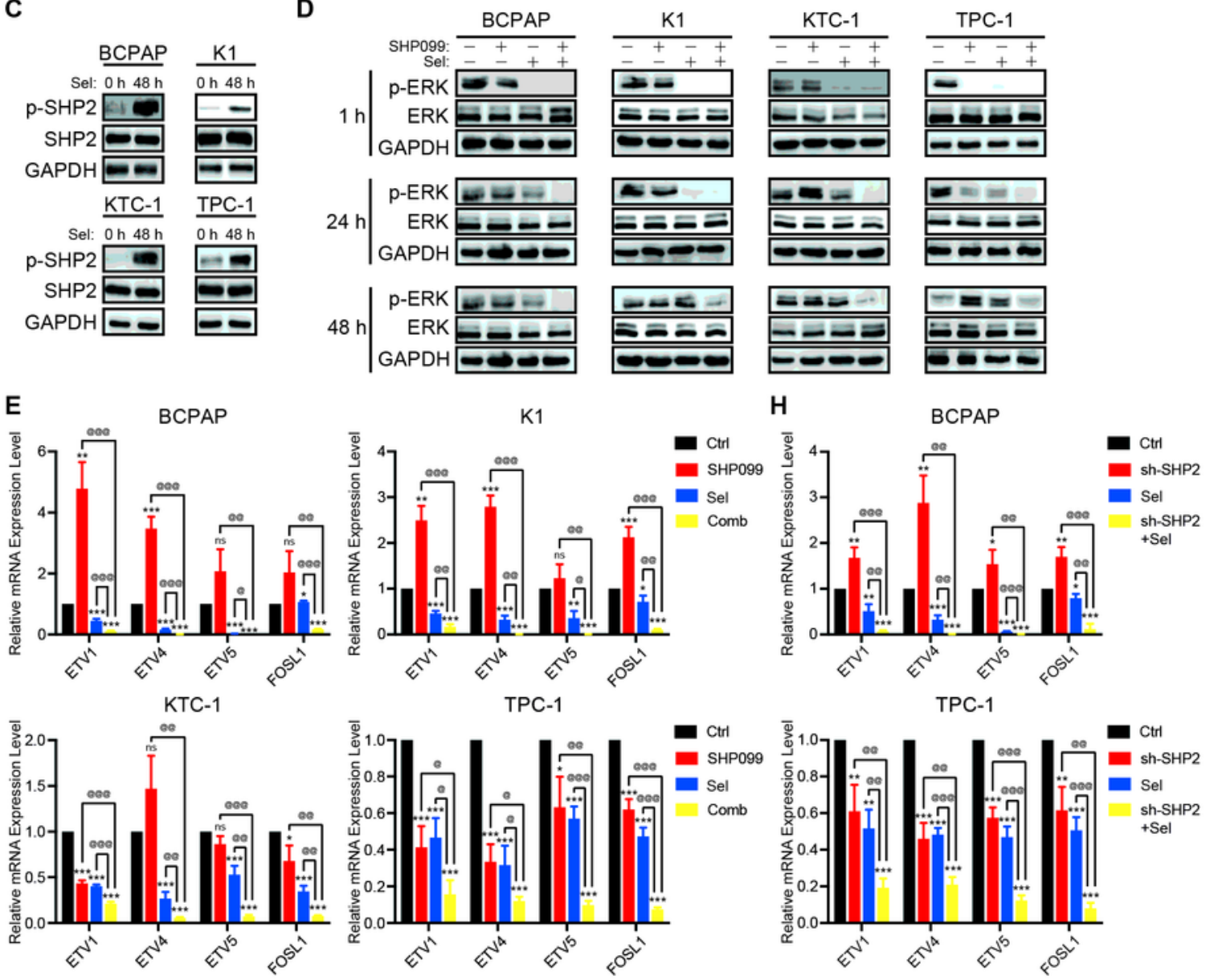

TPC-1

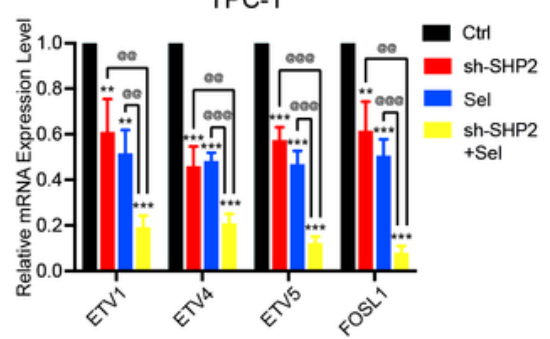

G

BCPAP

TPC-1

$$
\begin{aligned}
& \text { BCPAP TPC-1 } \\
& \text { sh-SHP2: } \frac{\text { BC }}{-++} \\
& \text { SHP2 }-- \\
& \text { P-ERK }-=-= \\
& \text { ERK } \\
& \text { GAPDH }
\end{aligned}
$$
empty vector sh-SHP2 empty vector sh-SHP2 Sel: 0h 1h 24h 48h 0h 1h 24h 48h 0h 1h 24h 48h 0h 1h 24h 48h SHP2 p-ERK

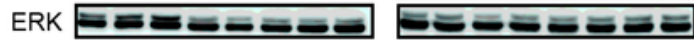

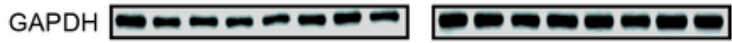




\section{Figure 3}

SHP099 abrogated the RTKs-induced rebound of the ERK pathway in PTC cell lines with 48h MEKi treatment. (A) The western blot revealed the ERK reactivation in 4 PTC cell lines within 48h selumetinib $(1 \mu \mathrm{M})$ treatment. Representative images of one experiment are shown. (B) The phospho-RTK arrays revealed that multiple RTKs were activated in PTC cell line K1 after $48 \mathrm{~h}(1 \mu \mathrm{M})$ selumetinib treatment. ${ }^{*} \mathrm{P}<0.05,{ }^{*} \mathrm{P}<0.01,{ }^{* \star *} \mathrm{P}<0.001$, two-tailed t-test. (C) Western blot revealed the SHP2 activation level in PTC cell lines treated with or without selumetinib for $48 \mathrm{~h}$. (D) The ERK activation level was analyzed by western blot in four PTC cell lines treated with DMSO (Ctrl), SHP099 $(10 \mu \mathrm{M})$, selumetinib $(\mathrm{Sel}, 1 \mu \mathrm{M})$, or both drugs (Comb) within 48h. (E) The ERK-dependent gene (ETV 1, 4 , 5, and FOSL1) expression were assessed by qRT-PCR in 4 PTC cell lines that were treated as indicated. ${ }^{*} P<0.05,{ }^{*} P<0.01,{ }^{\star} * \star P<0.001$, compared with Ctrl, @P<0.05, @@P<0.01, @@@P<0.001, compared with Comb, two-tailed t-test. ( $\mathrm{F}$ and $\mathrm{G})$ Knocking down SHP2 expression exhibited the similar effect as the SHP099 treatment in BCPAP and TPC-1 cell lines. (F) Western blot analysis of SHP2 expression after knocking down SHP2 expression in BCPAP and TPC-1 cell lines. (G) Knocking down SHP2 expression similarly abolished the ERK rebound after 48 h MEKi treatment. $(H)$ The combination of selumetinib $(1 \mu \mathrm{M})$ and SHP2 knockdown suppressed the ERK-dependent gene (ETV 1, 4 and 5, and FOSL1) expression. All data were represented as mean \pm SD of three independent experiments. 


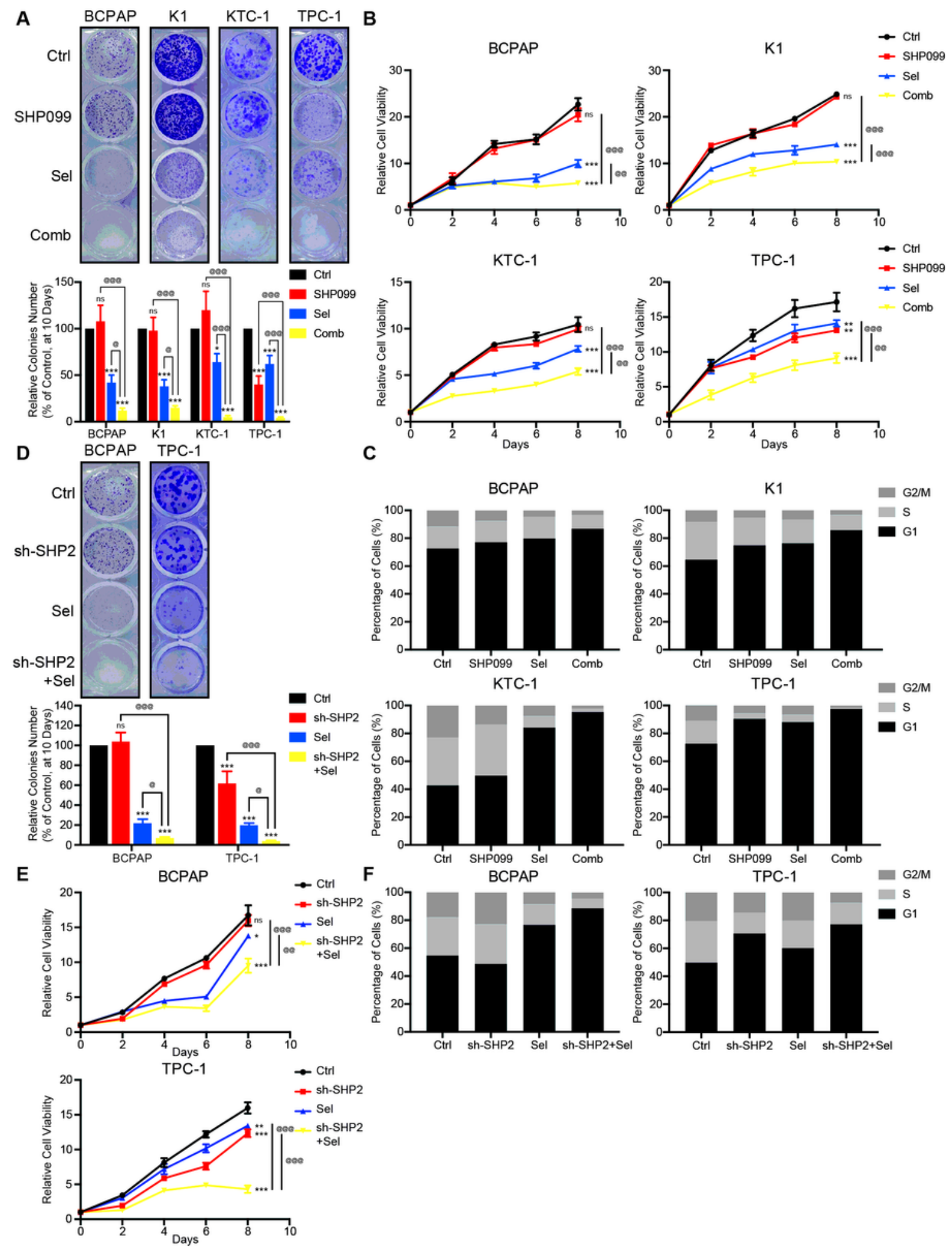

Figure 4

The combination of SHP099 and selumetinib exhibited synergy effect in PTC with various genetic backgrounds. (A and B) Four PTC cell lines were treated with DMSO (ctrl), SHP099 (10 $\mu$ M), selumetinib $($ Sel, $1 \mu \mathrm{M})$, or both drugs (Comb). The colony formation (A) and cell viability (B) assays were assessed at 10 and 0-8 days, respectively. ${ }^{*} \mathrm{P}<0.05$, ${ }^{\star} \mathrm{P}<0.01$, ${ }^{\star * * P}<0.001$, compared with $\mathrm{Ctrl}$, @P<0.05, @@P $<0.01$, @@@P<0.001, compared with Comb, ANOVA-test. All data were represented as mean \pm SD of three 
independent experiments. Compared with single agent, the SHP099/selumetinib combination group exhibited a more significant effect in inhibiting the progression in four PTC cell lines. (C) The SHP099/selumetinib combination significantly induced the G1 phase arrest in the four PTC cell lines. The cell cycle distribution was assessed by flow cytometry. The percentage of cells in the G1, S and G2/M phase was plotted. Colony formation assays (D), the cell viability assays $(E)$ and the cell cycle distribution (F) revealed that, knocking down SHP2 expression similarly exhibited the similar effect as the SHP099 treatment after combination with selumetinib.
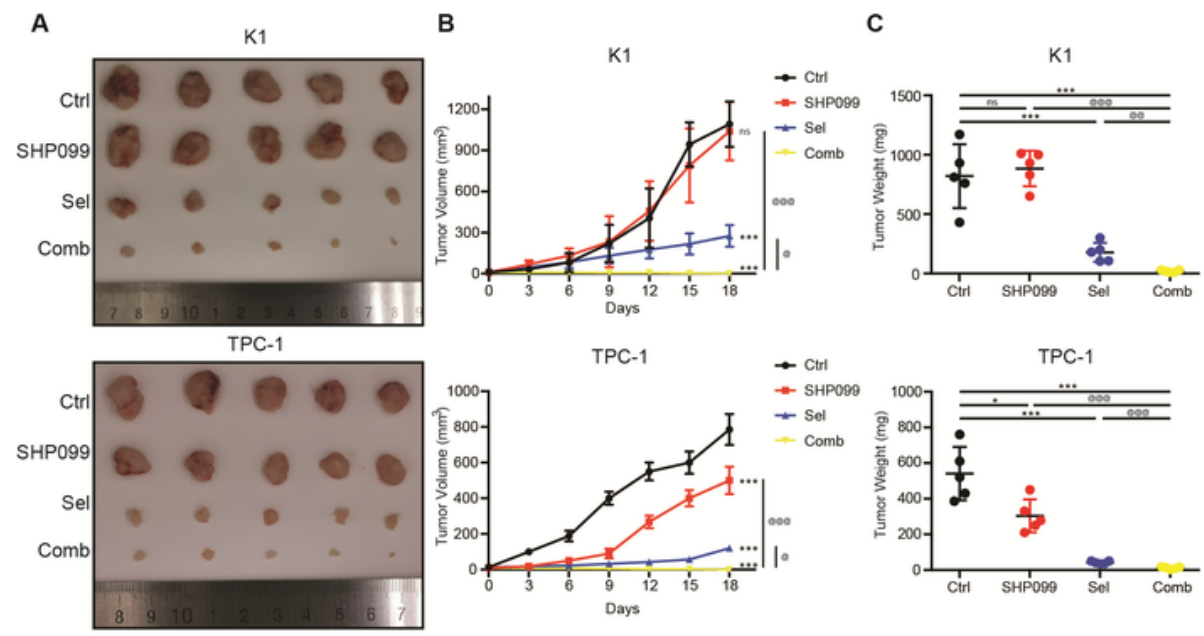

$$
\text { D }
$$
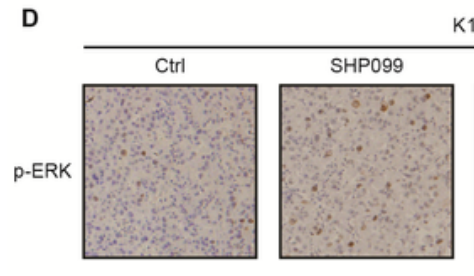

K1
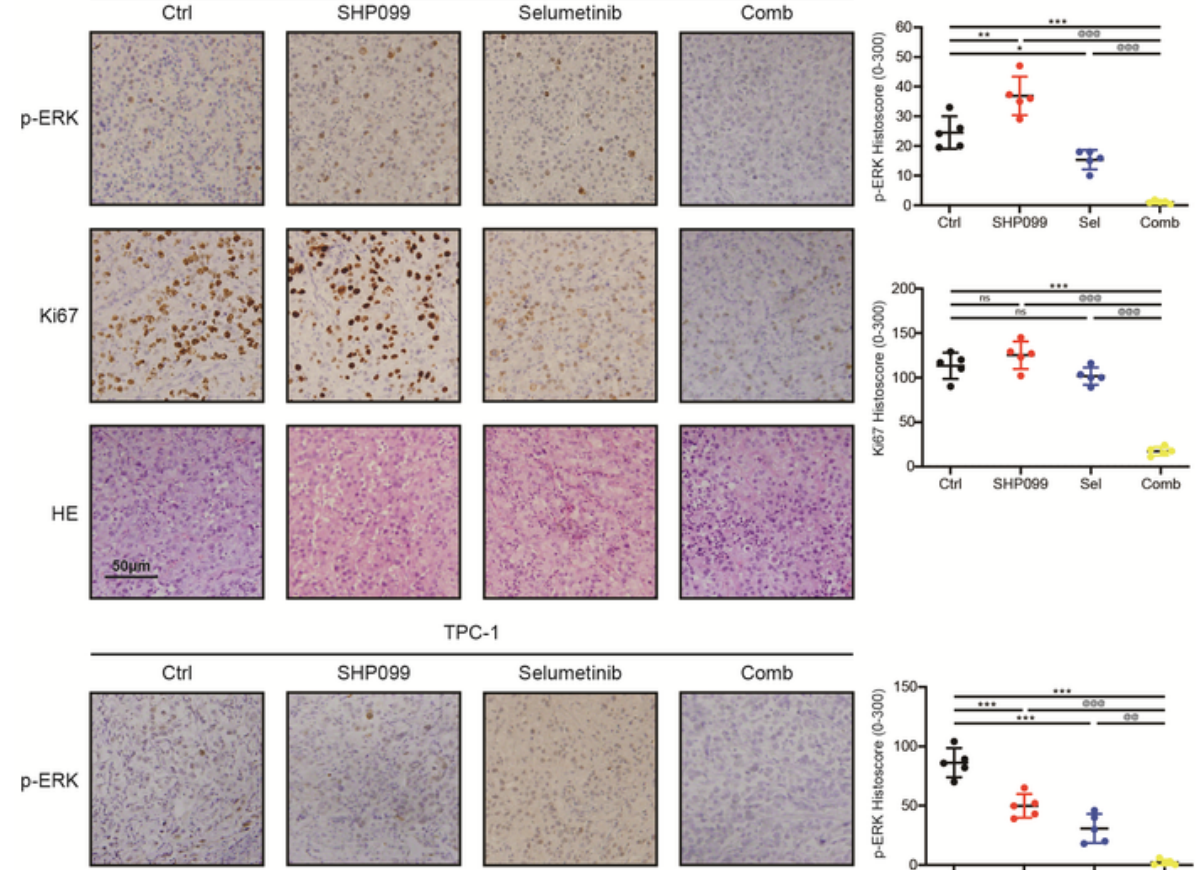

TPC-1
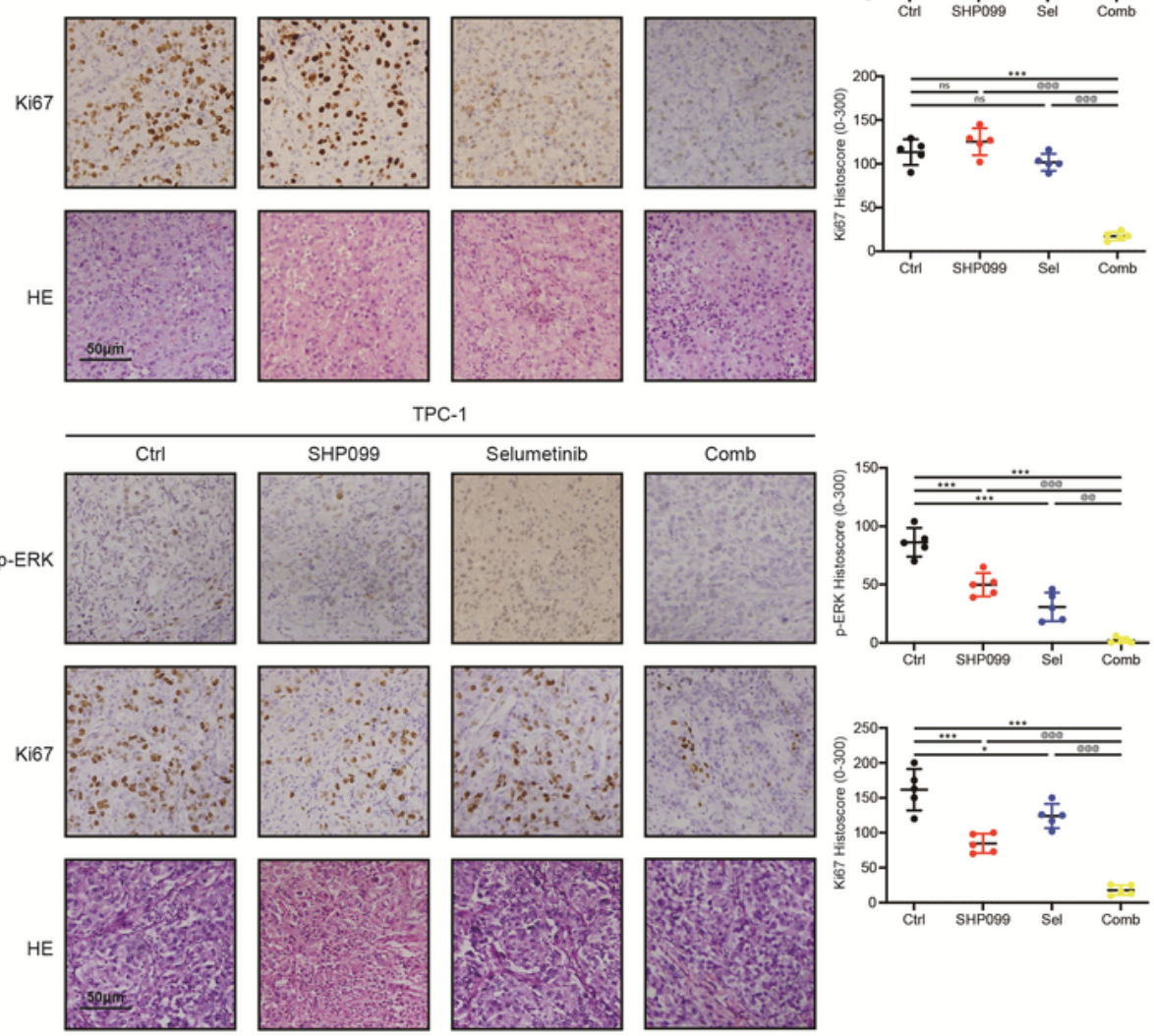


\section{Figure 5}

The combination of SHP099 and selumetinib was a promising therapeutic approach for advanced PTC in vivo. (A) Images of tumors dissected from NSG mice injected with the K1 and TPC-1 cells, respectively.

(B) The growth curves of K1 and TPC-1 xenografts after treatment with DMSO (Ctrl), SHP099, selumetinib (Sel) and both drugs (Comb). All xenograft tumor tissue volumes were measured every three days, until the 18th day. (C)The weight of the xenografts were analyzed after treatment for 18 days; $n=5$, per group, *P<0.05, **P<0.01, ***P<0.001, compared with Ctrl, @P<0.05, @@P< 0.01, @@@P<0.001, compared with Comb, ANOVA-test. (D) Representative images and QuPath quantitation of p-ERK and Ki67 immunostaining of xenografts as described above. ${ }^{*} P<0.05$, ${ }^{\star} * \mathrm{P}<0.01$, ${ }^{\star \star \star} \mathrm{P}<0.001$, compared with Ctrl, @P<0.05, @@P<0.01, @@@P<0.001, compared with Comb, ANOVA-test.

A

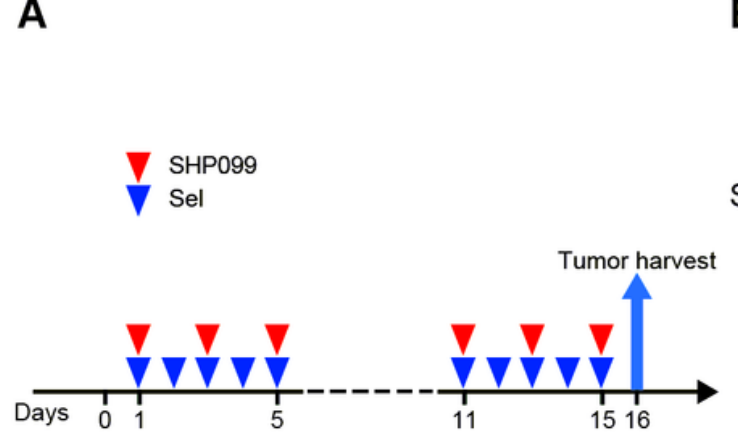

D
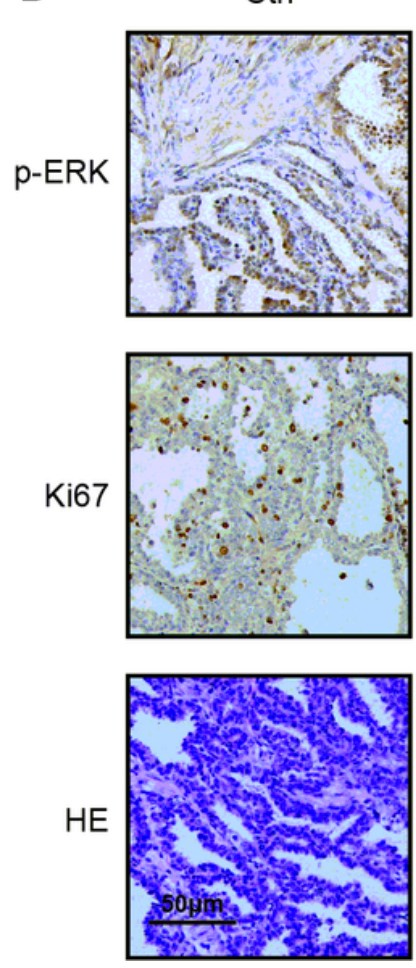

B

\section{Comb}

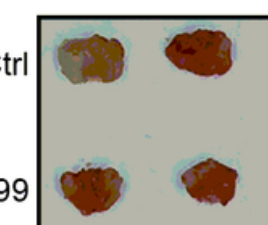

SHP099

Se

Sel
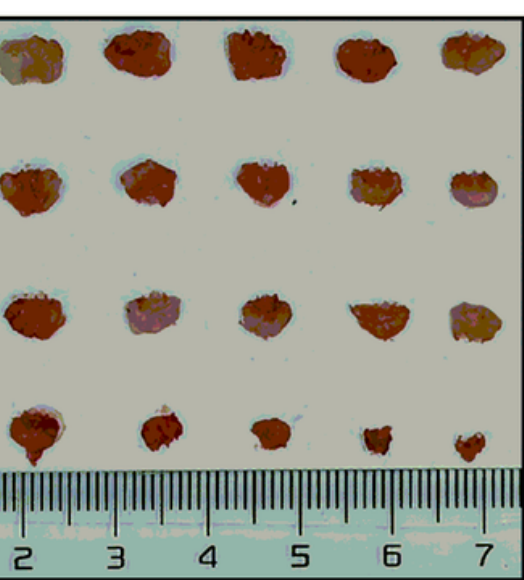

Selumetinib
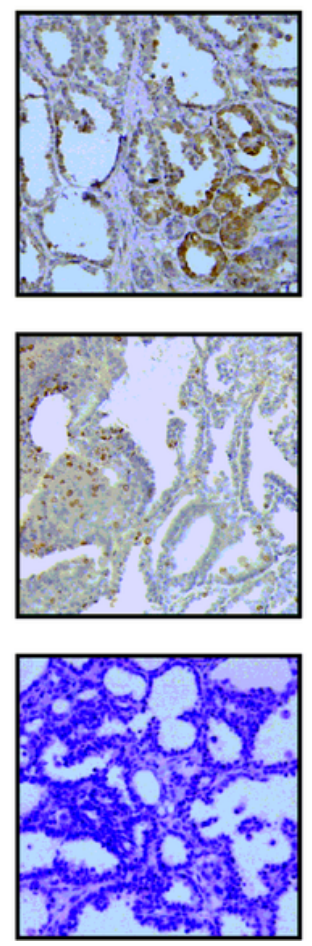
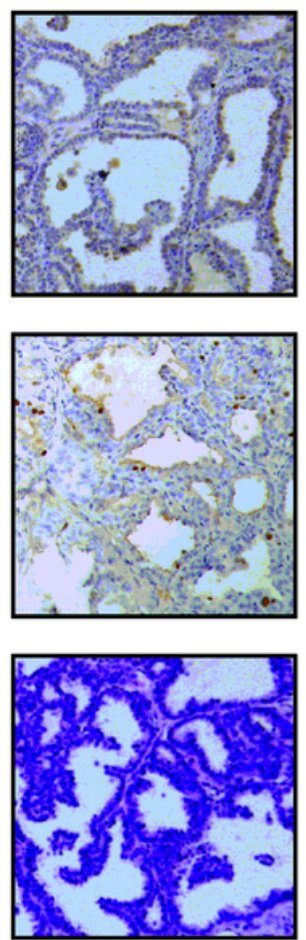

Comb
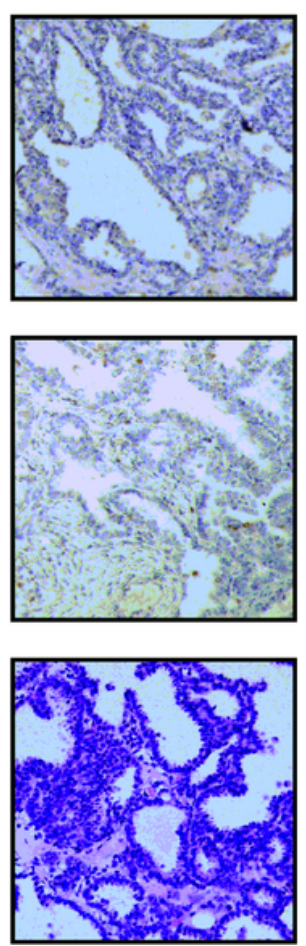

C

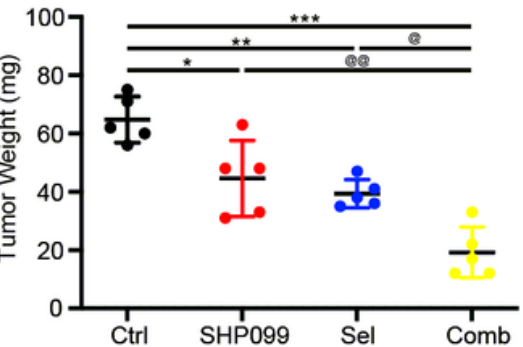

Figure 6 
The combined strategy was effective in the mice thyroid tumor models. (A) Representative images of mouse thyroid tumors. (B) Images of tumors dissected from thyroid tumor models. (C) The weight of the mouse thyroid tumors were analyzed after treatment for 18 days; $n=5$, per group, ${ }^{*} P<0.05,{ }^{*} P<0.01$, ***P<0.001, compared with Ctrl, @P<0.05, @@P<0.01, @@@P<0.001, compared with Comb, ANOVA-test.

(D) Representative images and QuPath quantitation of p-ERK and Ki67 immunostaining of the mice thyroid tumors as described above. ${ }^{*} \mathrm{P}<0.05,{ }^{*} \mathrm{P}<0.01$, ${ }^{\star \star *} \mathrm{P}<0.001$, compared with Ctrl, @P $<0.05$, @@P<0.01, @@@P<0.001, compared with Comb, ANOVA-test.

\section{Supplementary Files}

This is a list of supplementary files associated with this preprint. Click to download.

- GaoSupplementaryFigure3.tif

- GaoSupplementaryFigure2.tif

- GaoSupplementaryFigure1.tif

- GaoSupplementaryFigureLegends.docx 